\title{
Airway sensory nerves: a burning issue in asthma?
}

\author{
D Spina
}

\begin{abstract}
Asthmatic subjects cough and bronchoconstrict to various agents known to stimulate sensory nerves. A population of sensory nerves, the $C$ fibres, contain the neuropeptides substance $P$, neurokinin $A$ (NKA), and calcitonin gene-related peptide (CGRP). Capsaicin, the principal ingredient of hot peppers, selectively stimulates $C$ fibre afferents resulting in the release of these proinflammatory peptides. An upregulation in the function of sensory nerves may lead to augmented afferent and efferent function which, in asthma, could contribute to bronchial hyperresponsiveness, inflammation, and remodelling of the airway wall. Drugs specifically designed to attenuate the function of airway sensory nerves may prove useful in the treatment of asthma.

(Thorax 1996;51:335-337)
\end{abstract}

Keywords: asthma, sensory nerves, mechanism of action.

Asthmatic subjects are not unique in their ability to bronchoconstrict to spasmogens such as histamine and acetylcholine, although as a population they tend to lie at one end of the continuum. However, asthmatic subjects cough and bronchoconstrict to adenosine, bradykinin, sulphur dioxide $\left(\mathrm{SO}_{2}\right)$, sodium metabisulphite, and distilled water more readily than do nonasthmatics. Bradykinin and $\mathrm{SO}_{2}$ have been shown to stimulate neuropeptide-containing sensory nerves. Similarly, capsaicin is a selective stimulant of these nerves and has been used as a tool to investigate the function of sensory $C$ fibres. Indeed, the intradermal injection or inhalation of capsaicin and the consumption of chillies is often associated with a painful burning sensation as a result of the activation of sensory $\mathrm{C}$ fibres.

The increased sensitivity of the airways toward adenosine, bradykinin, $\mathrm{SO}_{2}$, sodium metabisulphite, and distilled water in asthma could reflect an increase in sensory nerve function. Current dogma suggests that sensory neuropeptides released from nerves play an important part in bronchoconstriction, oedema, mucus secretion, and vasodilatation. However, it is apparent that activation of sensory nerves in humans results in reflex coughing, bronchoconstriction, and mucus secretion with little evidence of oedema. ${ }^{1}$ While there is convincing evidence of an increase in the afferent function of sensory nerves, the evidence of an augmented efferent response has therefore yet to be established. However, neuropeptides can also regulate the function of inflammatory cells and immune cells. It is this efferent function of sensory nerves that may also contribute towards airway inflammation, bronchial hyperresponsiveness, and airway remodelling.

\section{Scientific basis}

A number of sensory nerves and receptors are present in the airways including mechanoreceptors which are supplied by myelinated fibres $(A \delta$ and $A \beta)$ and several non-myelinated $C$ fibres with endings that are loosely classified as polymodal. There are two main groups of airway epithelium receptors, the rapidly adapting (irritant) stretch receptors and the C fibre receptors. ${ }^{2}$ Activation of the irritant receptors in the airways can result in reflex coughing, bronchoconstriction, and tracheal mucus secretion. Irritant and $\mathrm{C}$ fibre receptors have been described in human airways and are distributed throughout the trachea and larger bronchi. The irritant receptors have myelinated projections within the vagus and non-myelinated terminals in the airways and are stimulated by mechanical stimuli, various gases including ammonia, $\mathrm{SO}_{2}$, and cigarette smoke, and by a number of inflammatory mediators including histamine and prostaglandin $\mathrm{F}_{2 x}$. Most of the vagal fibres that originate from pulmonary and bronchial $C$ fibre receptors are non-myelinated $\mathrm{C}$ fibre afferents and have cell bodies in the nodose and jugular ganglion. ${ }^{3}$ Activation of these receptors causes reflex coughing and bronchoconstriction (figure). An interesting finding is that reflex coughing and bronchoconstriction is mediated by different populations of sensory nerves. ${ }^{2} \mathrm{C}$ fibre receptors are generally less sensitive to mechanical stimulation such as changes in lung volume. They are excited by some inhaled irritants such as $\mathrm{SO}_{2}$ and by various inflammatory mediators including bradykinin, histamine, prostaglandins, and by capsaicin.

A population of sensory nerves contains a number of neuropeptides including substance P, NKA, and CGRP. ${ }^{4}$ Immunohistochemical 


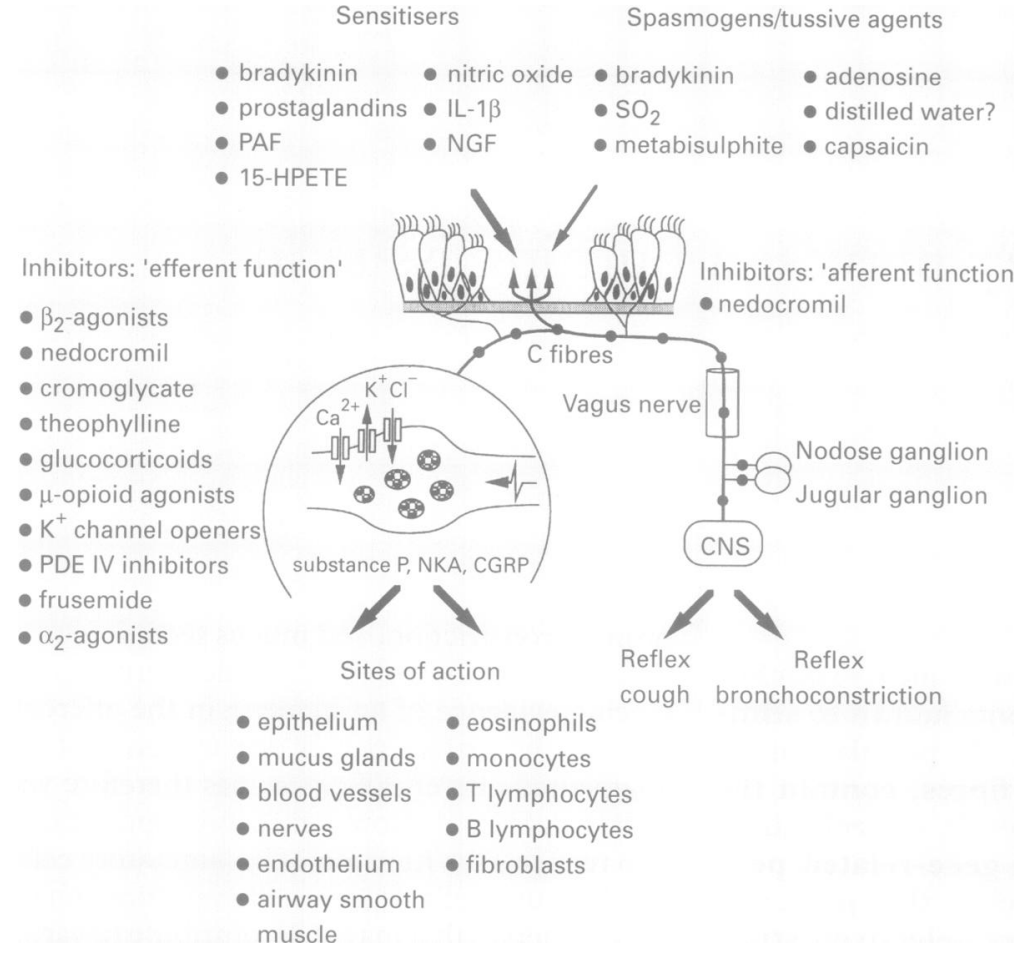

Diagrammatic representation of the innervation, function, and regulation of sensory nerves in the airways. Activation of sensory nerves by various spasmogens/tussive agents will lead to depolarisation of $C$ fibres (arrow) and the subsequent release of neuropeptides from nerve terminals (inset). Agents that lower the threshold for activation of sensory nerves (sensitisers) increase the ability of spasmogens/tussive agents to stimulate these nerves. $N K A=$ neurokinin $A ; C R G P=$ calcitonin gene-related peptide; $15-H P E T E=15-$ hydroperoxyeicosatetraenoic acid; $P A F=$ platelet activating factor; IL=interleukin; $N G F=$ nerve growth factor; $\mathrm{SO}_{2}=$ sulphur dioxide; $P D E=$ phosphodiesterase; $K^{+}=$ potassium; $\mathrm{Cl}^{-}=$chloride; $\mathrm{Ca}^{2+}=$ calcium. Afferent and efferent function refer to the sensory and effector function of sensory nerves, respectively.

techniques have shown that substance P-containing fibres are sparsely distributed within the bronchial epithelium, around blood vessels, bronchial smooth muscle, and local tracheobronchial ganglia. Autoradiographic and functional studies have confirmed the presence of neuropeptide receptors over a number of tissues including airways epithelium, submucosal glands, and nerves. ${ }^{5}$ In humans the sensory neuropeptides mediate contraction of the airway smooth muscle, mucus secretion, increase in nasal blood flow and oedema in the skin. ${ }^{5}$ Furthermore, neuropeptides can increase the expression of adhesion molecules on vascular endothelium and stimulate human $T$ and $B$ lymphocytes, monocytes, eosinophils, and fibroblasts. ${ }^{46}$ The neuropeptide substance $P$ has also been shown to increase airways responsiveness in asthma. ${ }^{6}$ Thus, neuropeptides released within the airway wall have the potential to stimulate inflammatory and immune cells (figure).

While it is apparent that the activation of sensory nerves serves as a protective mechanism against foreign insults, ${ }^{1}$ an upregulation in the function of these nerves might contribute towards the bronchial hyperresponsiveness, inflammation, and airway wall remodelling observed in asthma. ${ }^{7}$ In the skin inflammatory mediators such as bradykinin, 15-hydroperoxyeicosatetraenoic acid (15-HPETE), prostaglandins, platelet activating factor (PAF), cytokines including interleukin (IL) $-1 \beta$, and neurotrophins including nerve growth factor
(NGF) are known to sensitise sensory nerves resulting in a lowering of the pain threshold. ${ }^{78}$ It is also apparent that, in response to inflammatory stimuli, sensory nerve growth and increased synthesis of neuropeptides can occur. $^{48}$ Whether similar changes in sensory nerve function occur in the airways is not clear. However, bronchial hyperresponsiveness induced by PAF $^{9}$ and allergen ${ }^{10}$ is attenuated following chronic treatment with capsaicin which destroys sensory C fibres. These experimental studies suggest that the function of airway sensory nerves can be altered and contribute to bronchial hyperresponsiveness.

A number of studies support the view that sensory nerve function might be altered in asthma. Thus, asthmatic patients cough and bronchoconstrict to agents that have minimal or no effect in non-asthmatic subjects (figure). It is unclear whether substance P-containing nerves are more abundant in the asthmatic lung. However, a reduction in substance Plike immunoreactivity has been documented in asthma, ${ }^{11}$ suggesting the depletion of neuropeptide stores from sensory nerves. Indeed, this is consistent with an increase in the level of substance P-like immunoreactivity detected in the sputum and in bronchoalveolar lavage fluid of asthmatic subjects. ${ }^{6}$ These levels were increased further following an exacerbation of asthma. ${ }^{6}$ The increased release of neuropeptides may also be due to a concomitant reduction in neutral endopeptidase (NEP) activity, an enzyme that degrades neuropeptides. However, NEP activity is not reduced in patients with mild asthma.

\section{Therapeutic potential}

Sensory nerves might be considered appropriate targets in the treatment of asthma. The development of selective neuropeptide antagonists $^{4}$ and drugs that inhibit the activation of sensory nerves and/or release of neuropeptides ${ }^{5}$ could have potential as therapeutic agents. Indeed, the neuropeptide receptor antagonist FK-224 afforded significant protection against bradykinin-induced bronchospasm in asthmatic patients. ${ }^{12}$ Similarly, a number of anti-asthma drugs including nedocromil, sodium cromoglycate, and theophylline have beneficial effects against cough and bronchospasm induced by indirectly acting agents. ${ }^{5}$ Novel agents such as opioid receptor agonists, the loop diuretic frusemide, and type IV phosphodiesterase isoenzyme inhibitors can also attenuate the release of sensory neuropeptides (figure), possibly via an action on ion channels located on sensory nerves. ${ }^{45}$

\section{Conclusion}

An increase in the function of sensory nerves can lead to pain and inflammation, and a burning issue is whether sensory nerve function in the airways is augmented in asthma, thus contributing toward the increased sensitivity to cough and bronchoconstriction. Furthermore, the chronic release of neuropeptides in the airway wall may play an important part in the 
activation of inflammatory and immune cells which could lead to airway wall remodelling. Thus, drugs that target airway sensory nerves may prove useful in the treatment of asthma.

1 Karlsson J-A. A role for capsaicin sensitive, tachykinin containing nerves in chronic coughing and sneezing but not

2 Karlsson J-A. Saint'Ambrogio G. Widdicombe J. Afferent neural pathways in cough and reflex bronchoconstriction. f Appl Physiol 1988;65:1007-23.

3 Ellis JL, Undem BJ. Pharmacology of non-adrenergic, noncholinergic nerves in airway smooth muscle. Pulmon Pharmacol 1994;7:205-24. 4 Maggi CA. Tachykinins and calcitonin gene-related peptide ings of sensory nerves. Prog Neurobiol 1995;48:1-98.

5 Barnes PJ, Baraniuk JN, Belvisi MG. Neuropeptides in the respiratory tract. Part I. Am Rev Respir Dis 1991;144: $1187-98$.

6 Joos GF, Germonpre PR, Pauwels RA. Neurogenic inflammation in human airways: is it important? Thorax 1995;50:217-9.
7 Adcock JJ, Garland LG. The contribution of sensory reflexes and 'hyperalgesia' to airway hyperresponsiveness. In: Page CP, Gardiner PJ, eds. Airway hyperresponsiveness: is it really important for asthma? Oxford: Blackwell Scientific, 1992: 234-55.

8 Dray A, Urban L, Dickenson A. Pharmacology of pain. Trends Pharmacol Sci 1994;15:190-7.

9 Spina D, McKenniff MG, Coyle AJ, Seeds EAM, Perretti $\mathrm{F}$, Tramontana $\mathrm{M}$, et al. Effect of capsaicin on PAFinduced bronchial hyperresponsiveness and pulmonary cell accumulation in the rabbit. $\mathrm{Br} \mathcal{F}$ Pharmacol 1991;102: 1268-74.

10 Matsuse T, Thompson RJ, Chen X-R, Salari H, Schellenberg RR. Capsaicin inhibits airway hyperresponsiveness but not lipoxygenase activity or eosinophilia after repeated aerosolized antigen in guinea pigs. Am Rev Respir Dis 1991;144:368-72.

11 Lilly CM, Bai TR, Shore SA, Hall AE, Drazen JM. Neuropeptide content of lungs from asthmatic and nonasthmatic patients. Am ₹ Respir Crit Care Med 1995;151:548-53.

12 Ichinose $M$, Nahajima $N$, Takahashi $T$, Yamauchi $H$, Inoue $\mathrm{H}$, Takishima $\mathrm{T}$. Protection against bradykinin-induced bronchoconstriction in asthmatic patients by neurokinin receptor antagonist. Lancet 1992;151:1248-51. 